\title{
GNC Design for Asteroid Orbit Modification Missions
}

\author{
Miguel Hagenfeldt ${ }^{1}$, Juan L. Cano ${ }^{2}$ and Luis F. Peñin ${ }^{3}$ \\ Elecnor Deimos. Tres Cantos. Madrid 28760, Spain \\ Claudio Bombardelli ${ }^{4}$ and Jesús Peláez $z^{5}$ and \\ Technical University of Madrid, Madrid 28040, Spain \\ and \\ Eleonora Luraschi ${ }^{6}$ and Andrés Gálvez ${ }^{7}$ \\ ESA / ESTEC, 2200 Noordwijk. The Netherlands
}

\begin{abstract}
This paper shows the GNC design process for a mission aiming to find, approach and modify the orbit of a small asteroid. A large number of such bodies are orbiting the Sun in our Solar System, and some of them might become a real threat for the Earth during next few decades. While the size of the asteroid, besides its orbit, greatly determines the attention that should be given to any specific body, it is also necessary to start developing the concepts and technologies required to face these kind of challenges. For this purpose, these small asteroids represent a good training opportunity, since they allow testing the intended orbit modification principles with reasonable mission/system needs and with no impact risk for the Earth.
\end{abstract}

\section{Nomenclature}

$r \quad=$ asteroid orbit radius

$d \quad=$ asteroid reference diameter

$G \quad=$ asteroid slope parameter

$N_{A} \quad=$ no. of boxes in alongtrack direction

$N_{C} \quad=$ no. of boxes in crosstrack direction

$N_{R} \quad=$ no. of boxes in radial direction

$A=$ uncertainty region size in alongtrack direction

$C=$ uncertainty region size in crosstrack direction

$R=$ uncertainty region size in radial direction

$\varepsilon=$ cuboid dimensions in along- and crosstrack directions

$\lambda=\quad=$ cuboid dimensions in radial direction

$\beta \quad=$ maximum viewing angle

$f \quad=$ camera field-of-view

$p \quad=$ percentage of linear overlap between images

$N_{I} \quad=$ no. of images required for asteroid search sweep

\footnotetext{
'Senior GNC Engineer at Aerospace Engineering BU. Ronda de Poniente 19. Tres Cantos. Madrid 28760, SPAIN.

${ }^{2}$ Division Head at Aerospace Engineering BU, Ronda de Poniente 19. Tres Cantos. Madrid 28760, SPAIN.

${ }^{3}$ Division Head at Aerospace Engineering BU, Ronda de Poniente 19. Tres Cantos. Madrid 28760, SPAIN.

${ }^{4}$ Senior Researcher, Technical University of Madrid. Pza. Cardenal Cisneros 3. Madrid 28040, SPAIN

${ }^{5}$ Dept. Head, Technical University of Madrid. Pza. Cardenal Cisneros 3. Madrid 28040. SPAIN

${ }^{6}$ System Support, GSP Unit, D/PPC-PF, ESA Headquarters, Paris, FRANCE

${ }^{7}$ System Support, GSP Unit, D/PPC-PF, ESA Headquarters, Paris, FRANCE
} 


\section{Introduction}

W

HEN dealing with operations around a very small 2 to $4 \mathrm{~m}$ size Near Earth Object (NEO) (that is, more than three orders of magnitude fainter than Itokawa, the smallest asteroid ever visited by a spacecraft) the analysis and design of an effective terminal and proximity guidance and navigation strategy becomes paramount. The asteroid, whose orbit will likely be much more uncertain than Itokawa's, will need to be found by the S/C by imaging it against a star background (with a lightweight optical navigation camera or a more sophisticated imaging device). This analysis is presented in this paper to determine the navigation camera requirements, scanning strategy, terminal guidance and control activities and the required strategies.

After asteroid detection, achieved at a distance of some tens of thousands of $\mathrm{km}$, the satellite is manoeuvred to reduce its distance to the asteroid up to an indicative value of some tens of $\mathrm{km}$, at which a so-called Close approach sub-phase starts. During this phase, distance is further reduced in a controlled manner, while information about the asteroid is collected in order to characterize its orbit, shape and dynamics. These models shall be mandatory in the control of the satellite motion around the asteroid, during the so-called Close-proximity operations, during which the orbit modification really takes place.

This paper presents the GNC design associated to a mission based on the "Ion Beam Shepherd" (IBS) concept $\mathrm{t}^{1-3}$, a novel use of space propulsion in which the linear momentum carried by an accelerated plasma or ionic flux produced on board of a spacecraft (the "shepherd") is transferred to a macroscopic orbiting object (the "target") to modify its orbital and/or attitude dynamics for a variety of purposes including, but not limited to, active space debris removal and asteroid deflection.

Contents of the paper are as follows: after a first presentation about the IBS concept/fundamentals and mission requirements/analysis, the GNC needs for the asteroid search and hovering phases are analyzed with particular attention on relative navigation sensors and strategies. It includes GNC functional architecture and modes design, showing the relationship between the ion beam thruster design parameters and its ability for modifying the orbit of an asteroid of a given size. The paper concludes with a discussion on GNC programmatics and technology roadmap.

\section{Ion beam sheperd concept and fundamentals}

The ion beam shepherd concept (IBS) is a novel use of space propulsion in which the linear momentum carried by an accelerated plasma or ionic flux produced on board of a spacecraft (the "shepherd") is transferred to a macroscopic orbiting object (the "target") to modify its orbital and/or attitude dynamics. The flux can be generated using conventional electric propulsion systems like gridded ion thrusters (emitting an ionic beam) or space plasma thrusters (emitting a plasma beam) as the primary source of ionic/plasma flux that must be correctly pointed towards the target surface. Based on the divergence of the flow, which should be as small as possible, the position and/or attitude of the target can be controlled with the shepherd positioned at sufficient distance with minimum loss of efficiency due to partial overlap of the flux with the target envelope. In order to keep this distance constant the reaction of the ionic/plasma flow on the shepherd will need to be compensated by a secondary thruster pointed in the opposite direction. The key advantage of the method comes from its contactless nature: it does not require any solid mechanical interaction between the shepherd and the target object. Furthermore, because the beam pressure is distributed across the whole target cross-section the method is insensitive to the shape and aggregation state of the target. The method was first proposed and patented by the Technical University of Madrid in March 2010, and is depicted in Figure 1.

Using an asteroid orbit modification tool the IBS concept has been compared ${ }^{4}$ to another well known contactless asteroid deflection method whose applicability is being considered by space agencies and international research organizations: the gravity tractor (GT). Crucially, and unlike the GT, the IBS can provide a deflecting force whose

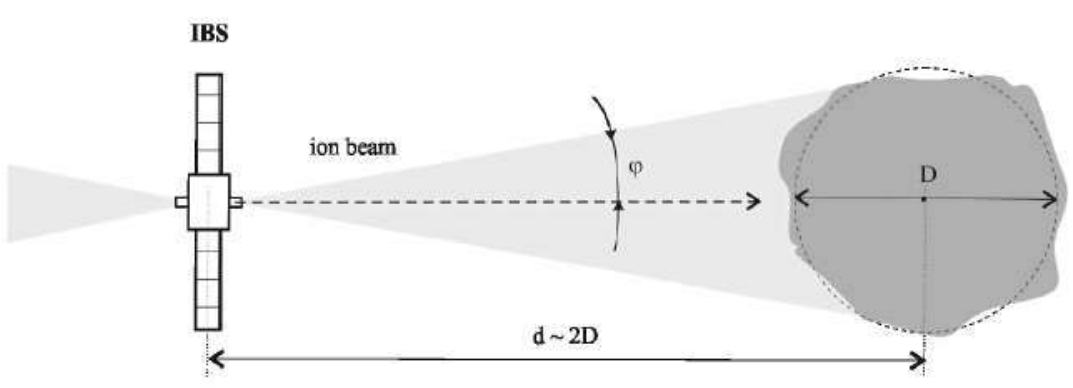

Figure 1. Schematic of ion beam shepherd asteroid actuation magnitude does not depend on the asteroid mass and can be employed with very small asteroids. As these are easier to deflect, more abundant and easier to reach with relatively low delta-V from Earth an IBS orbit modification demonstration mission may be carried out in a relatively short time span and with limited budget.

A key aspect of the IBS concept is the modelling of the ion/plasma beam expansion from the source up 
to the target surface. A smaller beam divergence is very desirable as it permits having the shepherd spacecraft located at a larger distance from the target hence reducing collision risks and minimising backsputtering. Typically there are two main factors determining the beam expansion: the thruster divergence at the beam source (determining the initial beam cone angle) and the thermal pressure of the neutralizing electrons (determining the rate of increase in beam divergence). The second factor is particularly relevant for the asteroid deflection case because the separation distances involved can become very large as the asteroid size increases.

The momentum and torque transferred from the plasma beam to the asteroid is given by the integral on the asteroid surface of the perpendicular momentum flux of the different species in the beam: single and double-charged ions, neutrals, plus the residual pressure of electrons and the momentum of sputtered material. The process of determining the forces and torques transmitted to a target can be complicated. This is due to the complex geometrical relation between the ion beam (whose velocity distribution follows a nearly Gaussian profile) and the surface of the target object. If the target can be approximated with a sphere simplified analytical expressions can be used. On the other hand, for the case of an object of irregular shape (as specified in the main mission requirements of the SEALS challenge) direct numerical integration is necessary. For this purpose, a numerical tool (Ion Beam Interaction Simulator IBIS) was developed, shown in Figure 2.

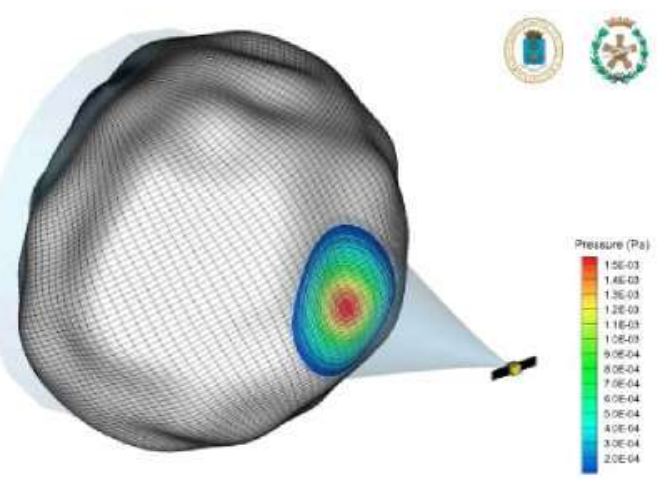

Figure 2. IBIS (Ion beam Interaction Simulator) software graphical output

\section{Mission requirements and analysis}

The mission requirements are explicited in this section, together with an overview of the mission analysis capabilities offered by Elecnor Deimos. This is a major input for subsequent work in the GNC/AOCS fields.

\section{A. NEO visibility and uncertainty box}

Small NEOs are often characterised by imprecise orbits as they are observable from Earth across relative short arcs. The accuracy in the knowledge of a small NEO orbit will strongly depend on its observation history and is described by its covariance matrix available, for instance, from the NEODyS ${ }^{5}$ or NEO JPL ${ }^{6}$ databases. When planning a future asteroid orbit modification mission one has to carry out an estimation of the evolution of the asteroid state transition matrix in the future by forward propagation in time and taking into account future measurements. A small NEO can only be observed from the ground when it is relatively close to the Earth and close to solar opposition (night-time viewing). The difficulty of performing a rendezvous with an asteroid of a few meters in size introduces new challenges that should not be underestimated. Crucially, a pre-planned rendezvous trajectory cannot be established because the NEO ephemerides are affected by uncertainties. For the purpose of prerendezvous asteroid searching, one needs to define a bounding box that has to be scanned by the spacecraft in search of the asteroid visual signature. The evolution in time of the size of this "uncertainty bounding box" has been computed using a small body propagator which includes the propagation of the orbit state transition matrix (using linear theory) to derive the corresponding covariance matrix in Cartesian coordinates, including the contributions of solar radiation pressure and Yarkovsky effect. A schematic definition and example results for a candidate NEO are shown in Figure 3.

When the asteroid is very faint the most efficient way to observe it is by having the spacecraft on a slightly lower orbit than the asteroid one and pointing its camera in the anti-solar direction towards the uncertainty ellipse. The relevant search region can then be conveniently defined as a prism with its faces parallel to the orbit velocity, the orbit radius and the orbit normal.

\section{B. Target NEO reference characteristics}

The contactless deflection of a small NEA and the measurement of such deflection is the ultimate goal of the main mission, and broken down requirements will need to be related to such goal. The GNC in question was developed assuming the following NEO reference characteristics:

- Orbit: the target asteroid orbit radius $\mathrm{r}$ must satisfy: $0.7 \mathrm{AU} \leq \mathrm{r} \leq 1.4 \mathrm{AU}$. In addition the orbit inclination must not exceed 5 degrees. 
- Impact risk: any risk for the asteroid to impact the Earth considering both its natural trajectory and its deflected one must be negligible.

- Mass: the reference mass for the target asteroid is set to 130 tons. This reference mass is used to impose a specific requirement on the deflection to be achieved, i.e. must be able to impart to an asteroid of 130 ton mass a deflection of $1 \mathrm{~m} / \mathrm{s}$.

- Shape and Morphology: an irregular object (i.e. not spherical) should be assumed, with a shape not known a priori. No assumption can be made on the aggregation state of the asteroid, which can range from a strong cohesive object to a rubble pile aggregate. The proposed deflection method will need to be sufficiently versatile to accommodate such variety of physical conditions.

- Size: the reference diameter $d$ for the target asteroid has to obey $2 \mathrm{~m} \leq \mathrm{d} \leq 4 \mathrm{~m}$.

- Material: a silicate asteroid is assumed, and a silicate surface is considered relevant for the ion-beam material interaction.

- Maximum Position Uncertainty: a reasonable limit for the size (twice the semi-axis and considering a 3-sigma ellipsoid) uncertainty bounding box is $500,000 \mathrm{~km}$ in the alongtrack direction (in the plane of the sky from the Sun) and $50,000 \mathrm{~km}$ in the radial direction from the Sun.
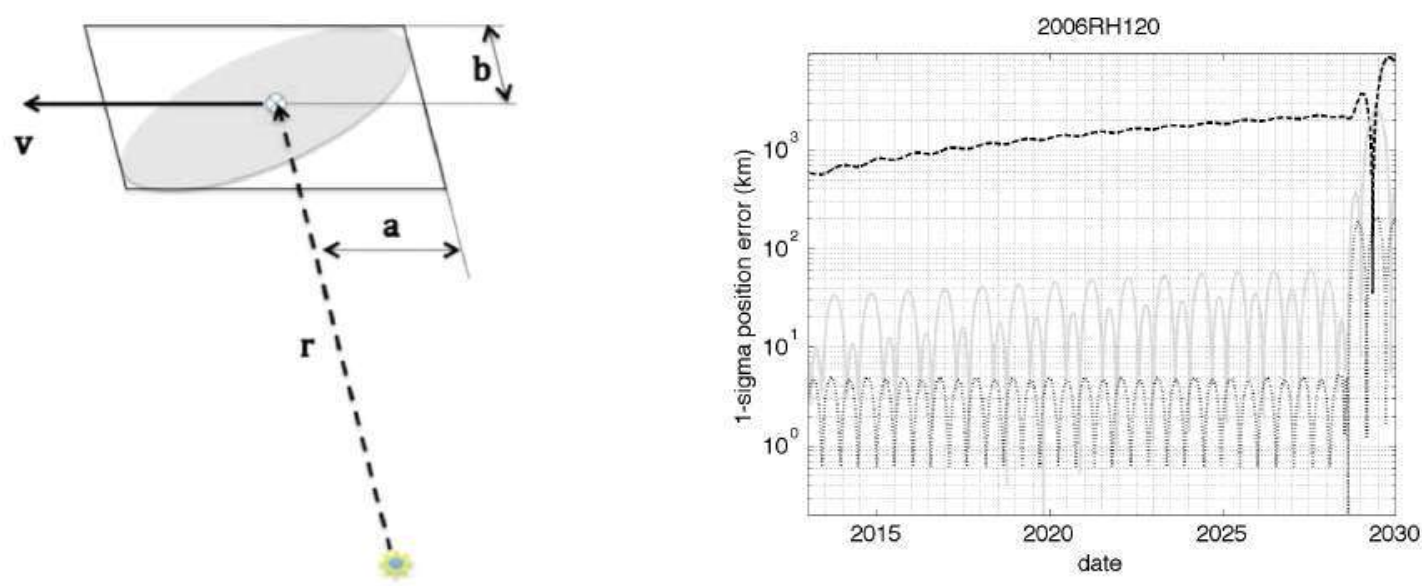

Figure 3. Left: schematic of uncertainty bounding box for the 1-sigma position uncertainty ellipsoid

(orbital plane projection) // Right: uncertainty bounding box for a candidate NEO

\section{NEO deflection requirements}

It is important to define deflection requirements that respect operational and technology constraints, e.g. exploiting a ground station radio link with the IBS spacecraft hovering at a few meters from the asteroid surface. The tangential velocity variation can then be determined by comparing the velocity of the propagated un-deflected asteroid trajectory with real-time velocity measurements taken during the deflection phase. The requirements are:

- Imparted delta- $V$ : capability to modify the NEO orbital speed (and hence semi major axis) by $1 \mathrm{~m} / \mathrm{s}$ in less than 3 years from launch of the mission. In the above relation one should note that the tangential delta- $\mathrm{V}$ variation is considered in the deflection requirement. This is because for the case of very small acceleration thrust (compared to local gravity) a tangential deflection maximises the variation of the semi major axis of the asteroid orbit, which is a very relevant deflection metric.

- Deflection manoeuvre duration: the total mission time is limited to 3 years that, depending on the duration of the other phases of the mission, will eventually set a limit on the maximum manoeuvre duration. After this consideration a maximum time of 6 months is allocated for the IBS phase.

- Delta-V measurement: the imparted delta-V has to be measured with sufficient accuracy (SNR $>5$ ).

- Spacecraft manoeuvrability during deflection: ensure a stable hovering, correct ion beam pointing and proper collision avoidance.

- Backsputtering contamination limits: maximum accumulated backsputtering thickness level of $200 \mathrm{~nm}$. This requirement may put a constraint on the minimum IBS-asteroid separation distance, also driven by the need for a safe collision avoidance capability. 
- Distance from Earth: a maximum distance from Earth of 0.5 AU during operation of the IBS.

- Redundancy. the spacecraft will need to embark four equal electric thrusters two of them acting as primary propulsion units and the remaining two as secondary. The same goes for the onboard computer and antennas.

\section{Interplanetary transfer requirements}

As the IBS phase will require of the use of low-thrust, it is sensible to assume that the same propulsion subsystem will be used for the transfer phase. Thus, it is certain that the propulsion trade-off for the transfer phase can already be solved by stating that the low-thrust system will be used both for the IBS phase and the transfer phase.

For the interplanetary transfer duration it is reasonable to allocate a maximum time of 2 years from launch to the start of the search phase. This will leave enough time for the asteroid search within its uncertainty region, the operation of the IBS phase and the characterisation and precise orbit determination phases. The limit for the duration of the asteroid search phase is set to 3 months and the duration of the IBS phase shall not last more than 6 months.

\section{E. Mission analysis}

This section presents an overview of in-house capabilities for analysis of transfer options and performances to the candidate asteroids. The analysis of transfers from Earth to the candidate asteroids has been carried out making use of a systematic branch and pruning algorithm. The algorithm has been used to find trajectories including multiple fly-bys. The pruning logic is based on the application of a set of filters aimed at achieving early elimination of non-feasible or poorly-performing trajectories. The branch and pruning algorithm is based on the calculation of chemical transfers, and a set of filters is employed to relate the feasibility and performance of such chemical missions to the equivalent low-thrust transfers. The following assumptions are typically considered:

- Launch opportunities in specific range of dates, and considering launcher constraints

- Maximum mission duration

- Low-thrust engine performances (used in filtering)

- Margin for delta-v computation: $25 \%$

- Earth flybys included in the search

- Arrival mass taken as performance index: this is not the only valid performance index, but is quite typical for an early selection phase as the current one.

Typical results are shown in Figure 4.
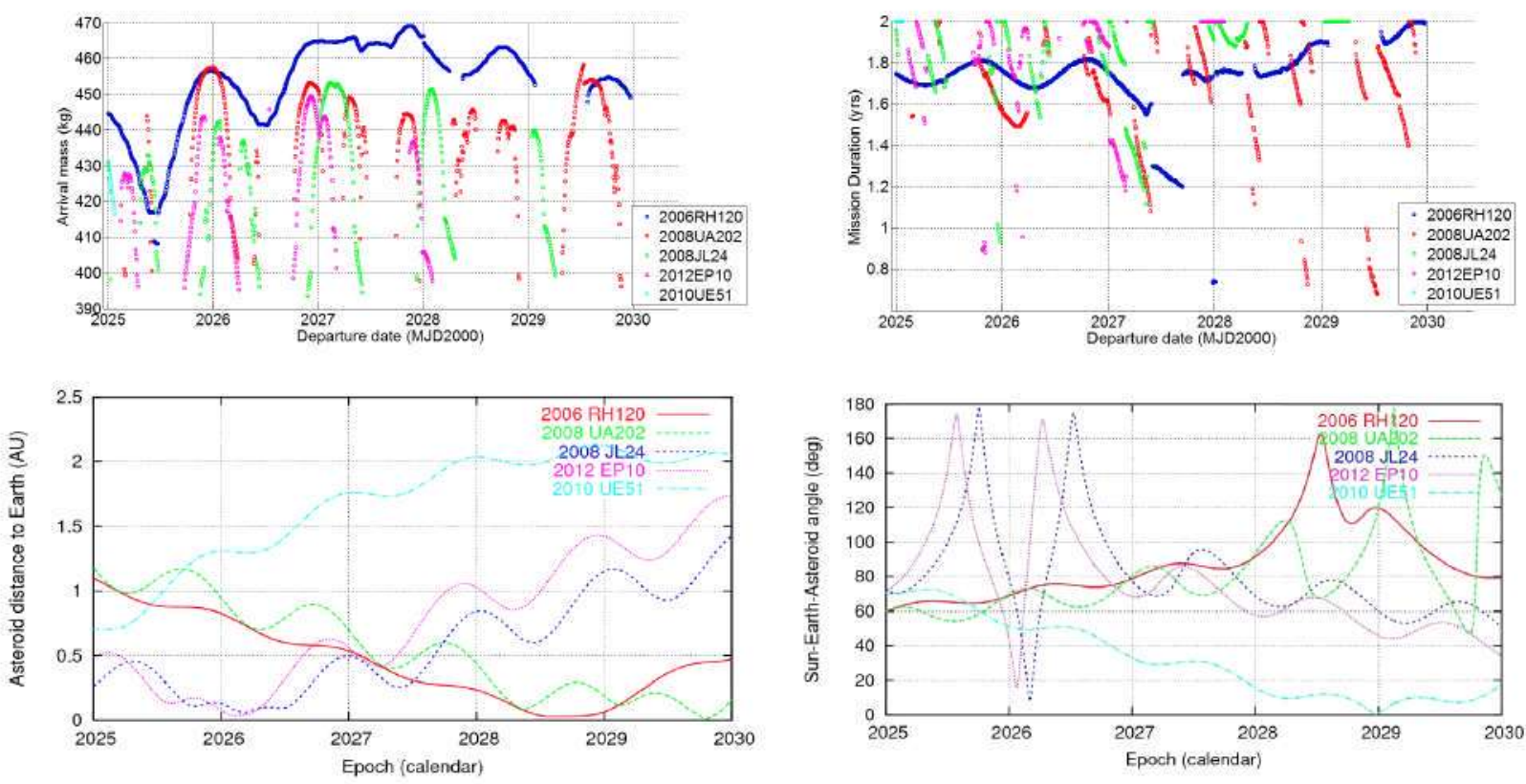

Figure 4. Mission analysis results for missions to a number of NEOs considered in MOSAIC study 


\section{F. Proposed mission timeline}

The results that are extracted from mission analysis results are used to find appropriate solutions for the several trade-offs the mission presents. These are not explicited here but gathered in the mission timeline presented in Table 1 and illustrated in Figure 5.

Table 1. Mission timeline for a NEO deflection mission

\begin{tabular}{|c|c|c|c|}
\hline Mission Phase & Start Event & Description & $\begin{array}{l}\text { Reference } \\
\text { Duration }\end{array}$ \\
\hline LEOP & $\begin{array}{l}\text { Departure of the launcher from the } \\
\text { pad }\end{array}$ & $\begin{array}{l}\text { The launcher takes the spacecraft and } \\
\text { possibly an additional propulsion stage } \\
\text { from the launch pad to injection in Earth } \\
\text { orbit }\end{array}$ & A few hours \\
\hline Escape phase & $\begin{array}{l}\text { Injection into escape orbit followed } \\
\text { by various perigee manoeuvres }\end{array}$ & $\begin{array}{l}\text { In case of direct escape after launch, this } \\
\text { phase does not exist. If an upper stage is } \\
\text { used to escape, this will bring the } S / C \text { to } \\
\text { achieve escape conditions by a set of } \\
\text { perigee manoeuvres }\end{array}$ & 2 weeks \\
\hline $\begin{array}{l}\text { Low-thrust subsystem } \\
\text { commissioning phase }\end{array}$ & End of last escape manoeuvre & $\begin{array}{l}\text { Operations are carried out to verify the } \\
\text { performances of the low-thrust engine. } \\
\text { Engine is thus tested in several } \\
\text { configurations }\end{array}$ & 90 days \\
\hline Nominal transfer phase & $\begin{array}{l}\text { Low-thrust subsystem declared } \\
\text { operational: engine switch on/s and } \\
\text { switch off/s, planetary swing-bys (if } \\
\text { foreseen) }\end{array}$ & $\begin{array}{l}\text { The low-thrust subsystem is operated in a } \\
\text { series of thrust and coast arcs to bring the } \\
\text { S/C to its target. Possibly, planetary swing- } \\
\text { bys are included in the transfer. }\end{array}$ & $\begin{array}{l}\text { As per } \\
\text { optimisation } \\
\text { process (max } \\
2 \text { years) } \\
\end{array}$ \\
\hline NEO search phase & Arrival to the NEO search region & $\begin{array}{l}\text { The NEO dispersion ellipsoid is swept in } \\
\text { the 3D space and the sky scanned in search } \\
\text { for the asteroid }\end{array}$ & 3 months \\
\hline Approach phase & Detection of the NEO & $\begin{array}{l}\text { The NEO is approached by the } \mathrm{S} / \mathrm{C} \text { down to } \\
\text { a safe distance }\end{array}$ & 2 weeks \\
\hline $\begin{array}{l}\text { Pre-IBS performance } \\
\text { phase }\end{array}$ & $\begin{array}{l}\text { Arrival to a certain close distance to } \\
\text { the NEO }\end{array}$ & $\begin{array}{l}\text { The } \mathrm{S} / \mathrm{C} \text { flights in formation with the NEO } \\
\text { in order to perform a preliminary } \\
\text { characterisation of the asteroid: precise } \\
\text { orbit, shape, rotation state, surface features, } \\
\text { etc. }\end{array}$ & 1 month \\
\hline IBS performance phase & Start of the IBS operation & $\begin{array}{l}\text { The operation of the IBS is carried out at } \\
\text { the defined operational distance }\end{array}$ & 6 months \\
\hline Post IBS phase & End of the IBS operations & $\begin{array}{l}\text { The } \mathrm{S} / \mathrm{C} \text { flights in formation with the NEO } \\
\text { in order to measure the achieved deflection } \\
\text { until this goal is achieved }\end{array}$ & 1 month \\
\hline $\begin{array}{l}\text { End of the nominal } \\
\text { mission }\end{array}$ & End of mission & & \\
\hline Extended mission & $\begin{array}{l}\text { End of the precise NEO orbit } \\
\text { estimation }\end{array}$ & $\begin{array}{l}\text { Activities could be the following: } \\
\text { - Landing on the asteroid } \\
\text { - Asteroid de-spinning experiment } \\
\text { - Asteroid push by contact method } \\
\text { - Increased IBS operation } \\
\text { - Flight to another asteroid }\end{array}$ & TBD \\
\hline $\begin{array}{l}\text { End of the extended } \\
\text { mission }\end{array}$ & End of mission & & \\
\hline
\end{tabular}



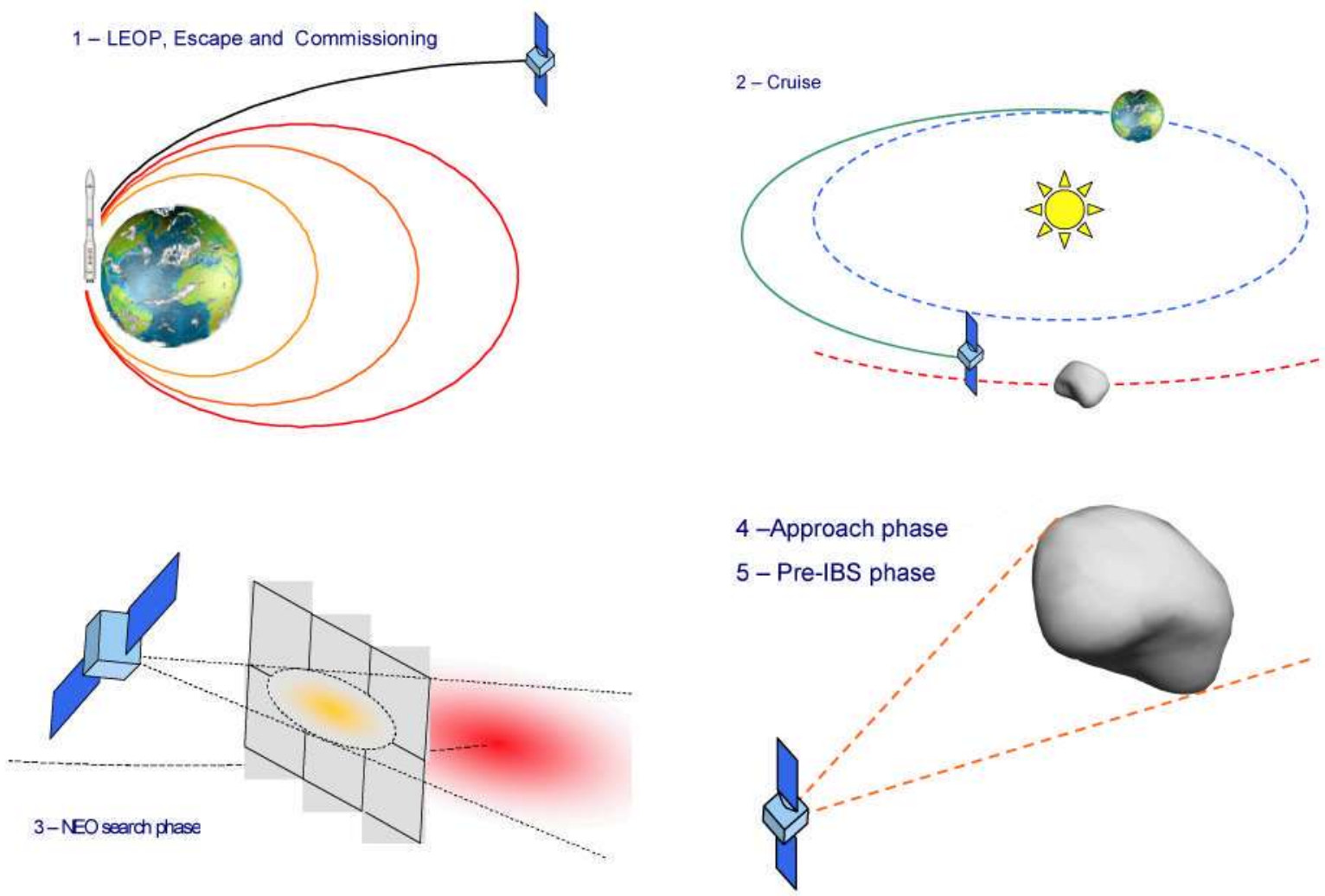

6 - IBS phase

7 - Post-IBS phase
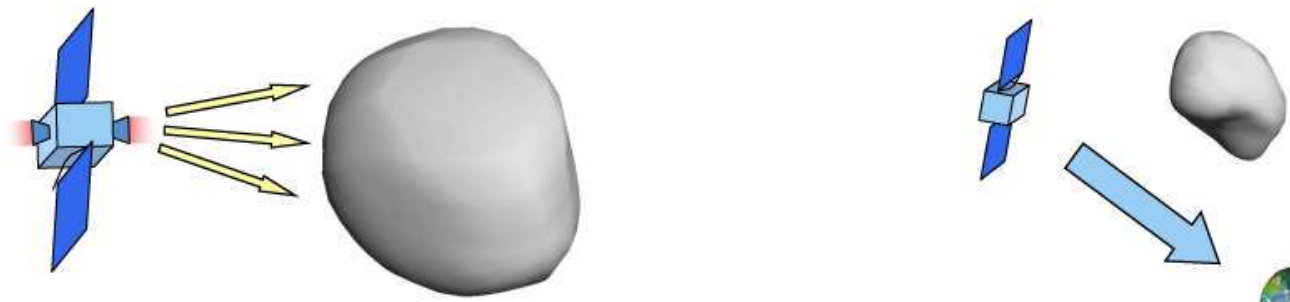

Figure 5. Mission phases for a NEO deflection mission using the IBS technology

\section{The asteroid detection challenge}

Due to the nature of the errors in the measurements taken on asteroid orbit, the uncertainty region in position is very elongated in the alongtrack direction, as discussed in Section III-A. Since the S/C arrival direction is close to that direction a reasonable search procedure is to sweep the uncertainty bounding box along the longest axis. This strategy was preliminary studied also for the Don Quijote mission ${ }^{7}$ but the targets of the present mission study are much fainter (typically 8-10 visual magnitudes fainter than the ones considered in the Don Quijote study). As a consequence, the scanning procedure for this case is much more challenging and has to be planned and optimized very carefully as it may have a strong impact on the mission design, complexity and cost.

The faint asteroid detection challenge shall be mitigated by several actions as:

1) Dimensioning an optical system and search strategy of higher performances, which shall have a large impact on the system design.

2) Selecting a target asteroid having ephemerides that are better known (high quality factors), which is very much favoured in those cases where observation periods are available just prior to the mission occurrence. 
Assuming the asteroid a uniform, perfectly diffusive Lambertian spherical surface for the calculation of its apparent magnitude, there is a geometrical locus of points with the same visual magnitude which would be the detection boundary for a given optical system. We have called such locus the detection polar and its representation is depicted in Figure 6. Curves are provided for cases where the difference between the absolute magnitude of the asteroid and the visual limiting magnitude of the optical device are respectively 13,15,17 and 19 magnitudes and the asteroid slope parameter is $\mathrm{G}=0.15$ (typical).

As expected, the maximum detection distance is obtained for a phase angle equal to zero, observing the typical brightness peak close to such angle. For increasing aspect angles, detection distance rapidly decreases. This suggests that a detection campaign can be composed of two processes:

1) A search procedure in position, by which the spacecraft sweeps the area occupied by the covariance ellipsoid

2) A scan procedure in orientation for a given position, by which the camera subsystem searches for the asteroid within the detection polar

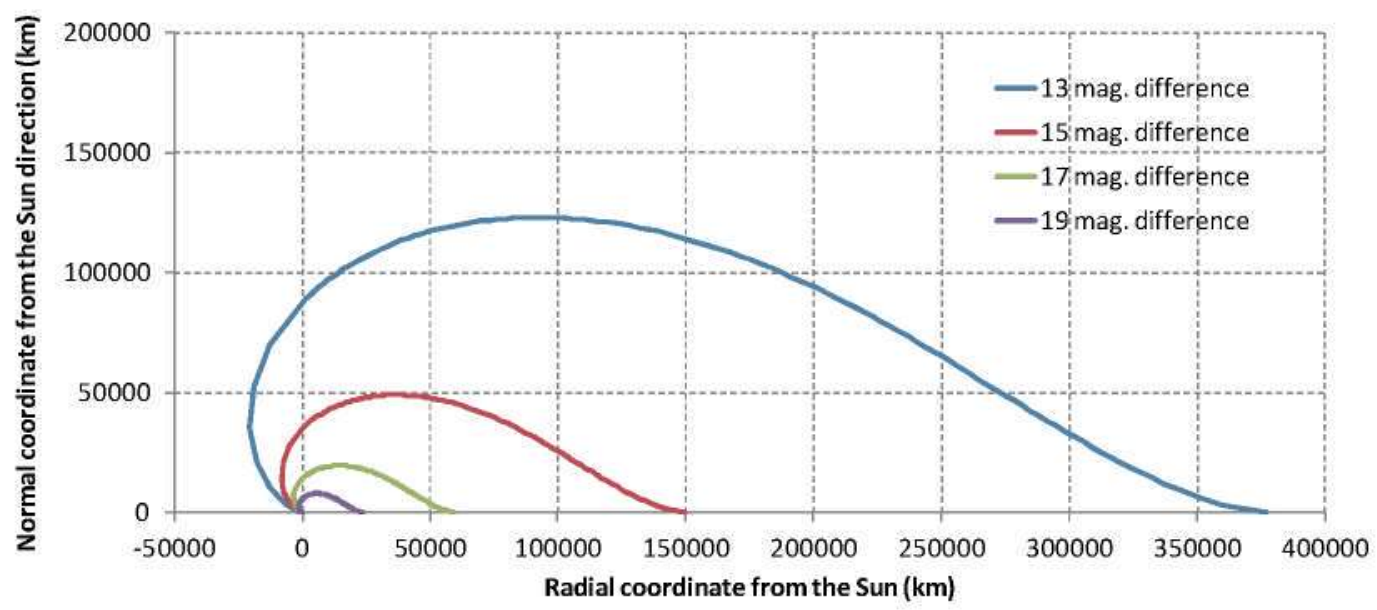

Figure 6. Detection polar for different values of the difference between asteroid absolute magnitude and the camera visual limiting magnitude (asteroid at $1 \mathrm{AU}$ from the $\mathrm{Sun}, \mathrm{G}=\mathbf{0 . 1 5}$ )

\section{G. Search procedure in position}

If the detection region is small compared to the uncertainty region, it is clear that a search procedure in position is required to try finding the asteroid in the uncertainty ellipsoid. The search procedure will then be characterised by a sweeping strategy of the uncertainty region which, for operational simplicity, will be approximated as a large rectangular box which shall contain the whole uncertainty region. By performing such approximation, the uncertainty region can be swept in a systematic manner by also assuming that the detection region (the one that can be covered by the spacecraft located at a given position) is modelled by a smaller box. Thus, the large uncertainty ellipsoid can be divided in smaller boxes (cuboids). The number of smaller boxes to

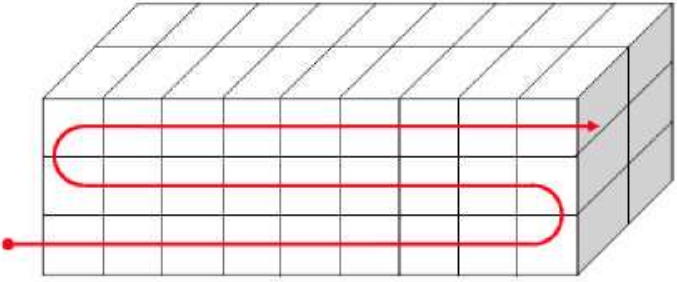

Figure 7. Preliminary sweeping strategy of the asteroid uncertainty cuboid sweep is $N=N_{A} X N_{C} X N_{R}$ equal to:

$$
N=\lfloor 1+\operatorname{int}(A / \varepsilon) \mid \cdot[1+\operatorname{int}(C / \varepsilon)|\cdot| 1+\operatorname{int}(R / \lambda)\rfloor
$$


where $A, C$ and $R$ are the uncertainty region dimensions in along-, crosstrack and radial directions, respectively, and $\varepsilon, \lambda$ the cuboid dimensions in the respective directions. Moreover, int \{\} is the integer rounding operator.

The total distance to sweep under such strategy would be:

$$
\left.D=\llbracket\left[\left(N_{A}+1\right) \cdot N_{C}-1\right] \cdot \varepsilon+\lambda\right] \cdot N_{R}
$$

The preliminary sweeping strategy of the NEO uncertainty box is depicted in Figure 7.

\section{H. Search procedure in orientation}

Having proposed an asteroid search strategy by sweeping the whole uncertainty region, one needs to know how to dimension the small search box. Assuming the S/C is located in one of the small search boxes and oriented to observe the space in the radial direction away from the Sun, it is necessary to find the asteroid within the detection polar. Assuming that for each search box we shall cover a maximum viewing angle $\beta$, the geometry depicted in Figure 8 can be used to determine the maximum cubic volume where visibility can be ensured. Such volume is the one contained by the detection polar within the $\beta$ angle. Such box provides the required values of $\lambda$ and $\varepsilon$ needed for search procedure in position (previous section). Being the actual polar a three-dimensional shape, the enclosure of a box within the $3 \mathrm{D}$ polar would require taking a dimension $\varepsilon / \sqrt{2}$ instead of just $\varepsilon$ if the box is equal in the alongtrack and crosstrack directions.

Once a value of the $\beta$ angle is selected for the mission, the scan process has to ensure that the selected solid angle is scanned by the S/C and the camera subsystem in search for the asteroid. Given a field of view (FoV) $f$ for the optical system and a percentage $p$ of linear overlap between images to ensure certain margin in the image processing, the required number of images to cover the solid angle shall be:

$$
N I=\left[1+\operatorname{int}\left(\frac{\beta}{f \cdot(1-p)}\right)\right]^{2}
$$

Figure 9 provides the number of images required to cover the solid angle for FoV of $5 \mathrm{deg}, 10 \mathrm{deg}$ and $15 \mathrm{deg}$, in an example geometry and target asteroid.

The proposed sweep strategy to search for the asteroid means that the relative motion of the S/C around the average asteroid orbit will need to be such that the S/C is forced to follow the specified path. This implies a limitation on the sweep speed which is the one that can be ensured with the $\mathrm{S} / \mathrm{C}$

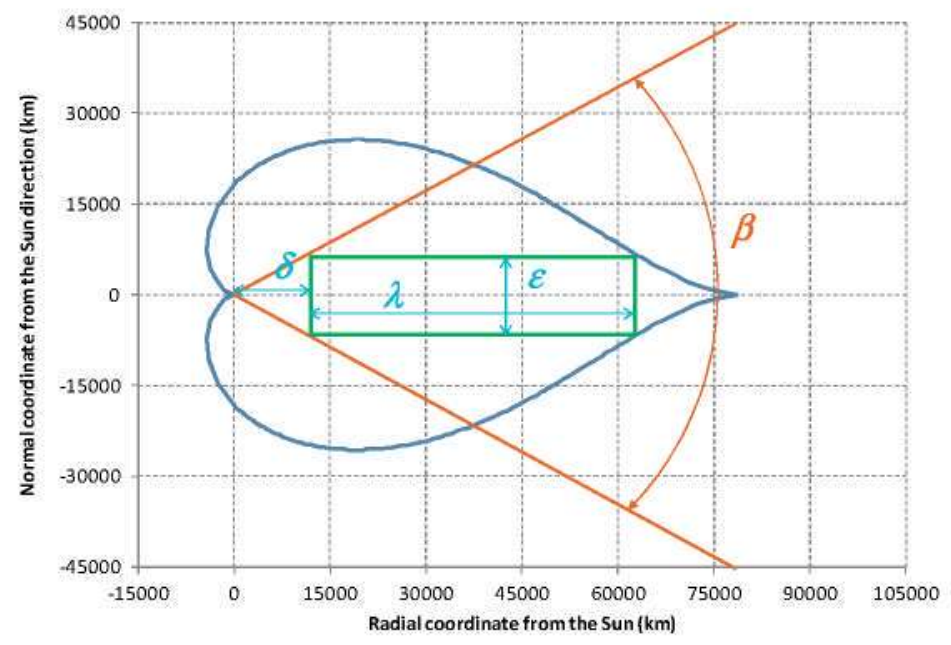

Figure 8. Detection polar for example absolute magnitude and camera limit and detection box for $\beta=60 \mathrm{deg}$ propulsion system and will also imply a certain fuel consumption and search period of time. Those parameters shall be also evaluated in order to determine the solution space of application for the design of the mission. For example, requirements on the following factors will help in constraining the definition of the system parameters:

- Maximum search time (e.g. 3 months)

- Maximum fuel consumption for search purposes (a given percentage of the boarded fuel)

- Minimum sweep velocity (related to the maximum search time but also depending on the maximum size of the uncertainty region and the available thrust modulus). 
For a forced drift in alongtrack direction, certain initial relative velocity wrt the asteroid can be maintained by pushing in radial direction, as shown in left side of Figure 10. It shall be assumed that the satellite possesses the intended relative drift velocity at the beginning of this phase, which can be obtained by proper targeting to this dynamics state at the end of the former transfer phase. The parameters to analyse are then:

- Drift velocity (i.e., km/day)

- Cost of low thrust profile (i.e. $\mathrm{m} / \mathrm{s}$ per $100,000 \mathrm{~km}$ )

- Maximum thrust needed to keep the intended drift velocity for a given satellite mass.

Analogously, in what regards the forced drift in radial direction it may be necessary to command a dedicated manoeuvre (right side of

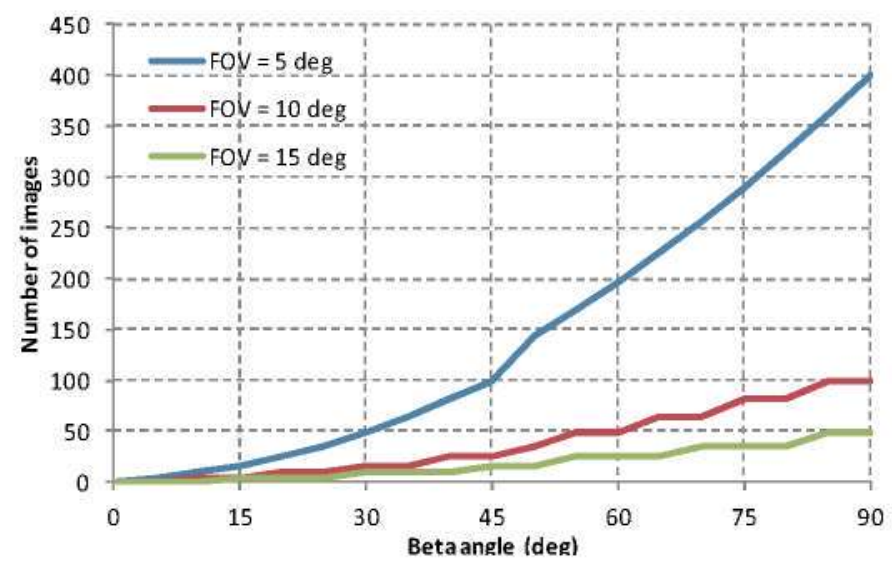

Figure 9. Number of required images to cover the detection box versus beta angle and camera $F o V$ Figure 10). The following conditions are assumed:

- The satellite starts at a null relative velocity with respect to the estimated asteroid orbit, since it is no longer valid to assume that initial relative velocity be provided by transfer manoeuvres

- The final intended state corresponds to a null relative velocity in radial direction, since the idea for this approach is to retreat (i.e., to go backwards to the initial position and velocity) after the complete length in radial direction is scanned

These manoeuvres are achieved using a terminal point guidance algorithm employed by Elecnor Deimos. In a project scenario, assuming $30 \mathrm{mN}$ of maximum thrust, it was found possible to scan up to $5,000 \mathrm{~km}$ in radial direction in less than 6 days.
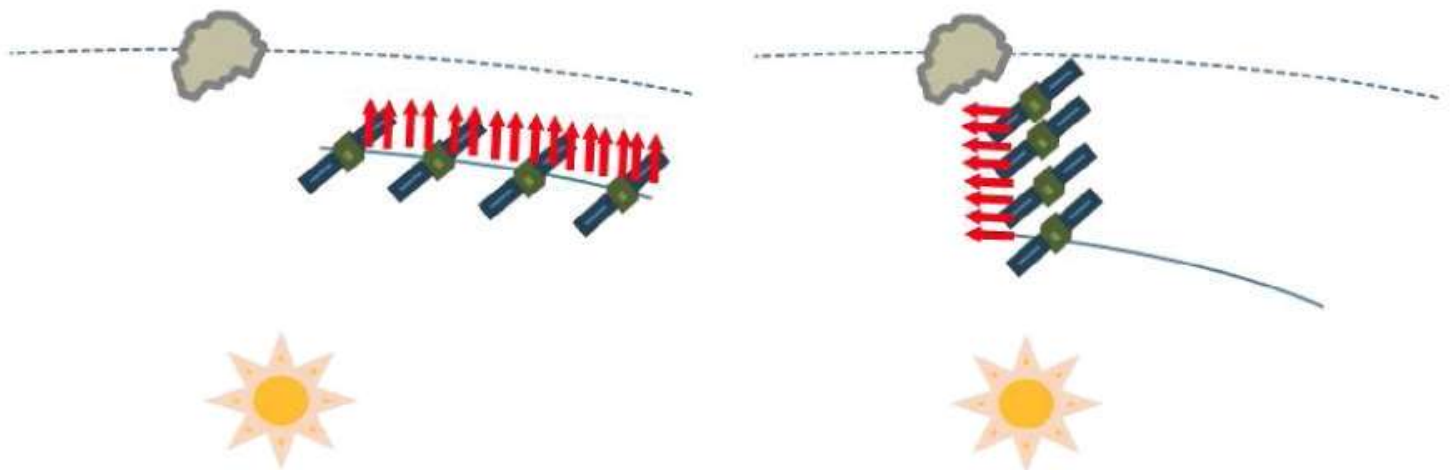

Figure 10. Forced, low thrust approach alongtrack (left) and in radial direction (right)

\section{Optimised search procedure and camera selection}

The optimised search procedure takes into account the methods described in the previous two sections, but in addition is based on the following aspects:

- It shall be preferred not to have to sweep the uncertainty region in the crosstrack direction, but just cover the uncertainty in such direction by an attitude scanning with the S/C taking images of the out-of-plane regions. This shall prevent from performing costly out-of-plane manoeuvres to sweep the ellipsoid. 
- It shall be preferred not to have to sweep the uncertainty region in the radial direction, but just have enough camera visual limit magnitude to cover the full uncertainty region dimension in the radial direction. This is certainly a constraining condition in the determination of the camera performances, as the detection polar shall then cover the whole uncertainty region in the radial direction, thus dimensioning the limit visual magnitude of the camera and/or the way the image processing is performed (e.g. multiple time integration concepts)

Accepting the above optimisations for the mission, it shall be possible to cover the full uncertainty region by just one pass in the alongtrack direction covering a large (e.g. 500,000 km) uncertainty region in such direction as represented in left side of Figure 11. Each of the smaller elongated parallelepipeds (right side of Figure 11) is the one contained in the detection polar presented in Figure 8. Within each of them, a scan in attitude will be performed to find the asteroid. Assuming a given radial distance $\lambda$ (e.g. $50,000 \mathrm{~km}$ ) to be covered and a given separation between the $\mathrm{S} / \mathrm{C}$ and the search region $\delta$ (e.g. $20,000 \mathrm{~km}$ ), one can extract (from Figure 6) the limit detection magnitude of the camera.
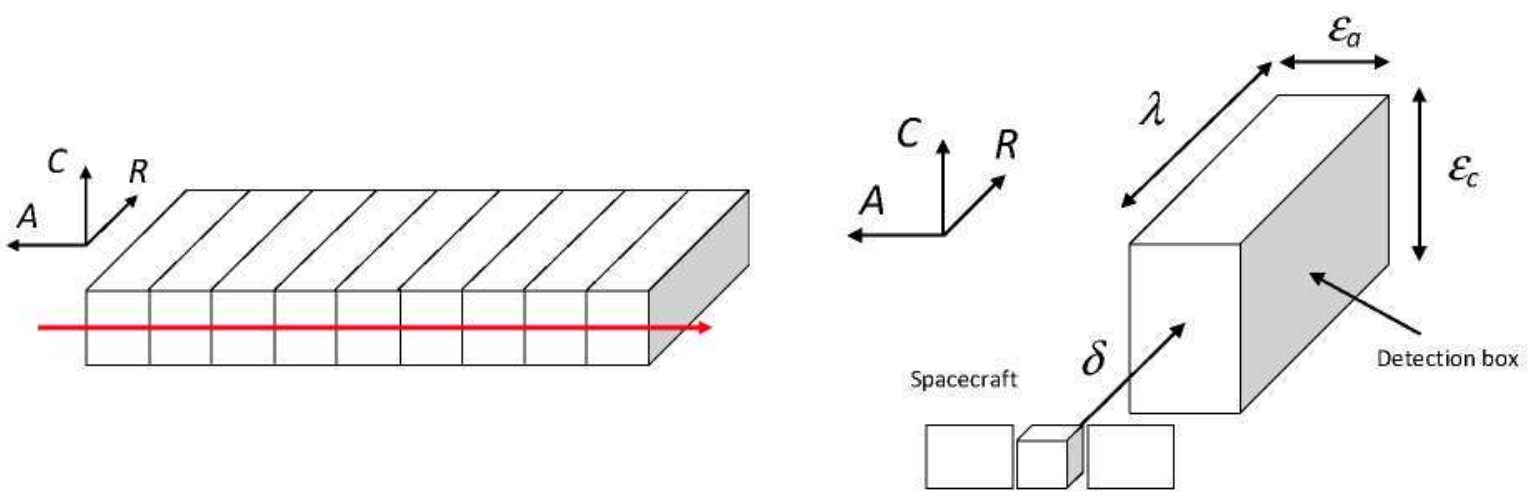

Figure 11. Optimised search strategy (left) and relative geometry between $\mathrm{S} / \mathrm{C}$ and detection box

For a specific mission project managed by Elecnor Deimos, a camera solution has been found suitable for the task of finding the target asteroids in three months and sweeping an uncertainty region with a maximum dimension alongtrack of $500,000 \mathrm{~km}$, crosstrack of $5,000 \mathrm{~km}$ and radial of $50,000 \mathrm{~km}$. It has been assessed that, under such conditions, a camera with a limiting magnitude of 13.5 should be enough to solve the detection of such small asteroids, provided the uncertainty region is scanned in the alongtrack direction in the available amount of time. The resulting constraints are well within the specification of COTS cameras for space applications and propulsion solutions for the $\mathrm{S} / \mathrm{C}$ sweeping process.

\section{In-orbit Operations Design}

\section{J. Overview}

Table 2 summarises the recommended asteroid proximity operations in a NEA deflection mission, between Approach and Post-IBS observation phases.

After asteroid detection, which shall be achieved within a maximum distance of less than sum of the delta and lambda parameters (e.g. $70,000 \mathrm{~km}$ ) described in Section IV, the thrusters shall be used to reduce the distance between the $\mathrm{S} / \mathrm{C}$ and the asteroid up to an indicative value of $50 \mathrm{~km}$, at which the Close approach sub-phase starts. Up to this point, for an asteroid with $4 \mathrm{~m}$ diameter, the body image will occupy almost one pixel in the (baselined) NAC. The S/C-asteroid distance is further reduced, while performing payload commissioning and calibration; the $\mathrm{S} / \mathrm{C}$ size in the NAC field of view grows up to approximately 10 pixels at this stage. For an asteroid of approximately 150 tons the Hill's radius in the asteroid-Sun system will be in the order of $500 \mathrm{~m}$; at this distance the asteroid will occupy up to approximately 100 pixels in the NAC, and the acquisition of information for the purpose of characterising the asteroid shape can be started.

At the end of the two-weeks long approach phase the Pre-IBS performance phase is started. At approximately $500 \mathrm{~m}$ from the asteroid, system tests to simulate the IBS functioning mode are executed, thus involving the simultaneous switch-on of both EP thrusters. Such tests are executed nominally outside the sphere of influence of 
the asteroid to reduce the risk of jeopardizing the mission with possible malfunctioning happening close to the attracting asteroid.

After the necessary system tests. the asteroid distance is reduced down to approximately $20 \mathrm{~m}$. Such close hovering position is reached along the Sun-asteroid line, in order to achieve maximum illumination conditions during the close characterisation phase, aimed at determining with precision the asteroid shape, its surface features and rotational state for the subsequent IBS phase operations. In this phase a coarse asteroid gravitational constant can be estimated. using the $\mathrm{S} / \mathrm{C}$ as test particle in the asteroid gravity field.

The coarse asteroid gravity estimation is mapped through the satellite motion in the vicinity of the asteroid, but noise contributions due to non-gravitational phenomena may limit the achievable accuracy and shall be taken into account. The gravimetric campaign will mainly be based on range and Doppler measurements during the experiment phase. combined with the elaboration of $\mathrm{S} / \mathrm{C}$-asteroid distance and asteroid size data. Such data shall be provided by ranging and imaging instruments. In addition, during the close characterisation phase the pre-deflection asteroid orbit determination will be carried out. Also for such purpose the elaboration of S/C-asteroid distance data will be necessary, thus meaning that the orbit determination campaign requires a maximum $\mathrm{S} / \mathrm{C}$-asteroid distance within the operational range of ranging sensors.

At the end of the Pre-IBS performance phase the S/C moves to acquire the nominal IBS position (thus alongtrack wrt the asteroid heliocentric orbit). Final system checks (if required) are executed, and the nominal IBS phase is started. At the end of the 3 months long IBS deflection phase (carried out at an indicative distance of 10 to $15 \mathrm{~m}$ from the asteroid surface), a safe distance to the asteroid is acquired, and the post-deflection asteroid orbit determination is executed to assess the effects of the ion beam shepherd phase.

If time avails. or at the beginning of a possible extended mission phase. additional observations of the asteroid surface features, shape, and rotational state could be acquired to assess the effects (e.g. characteristics of the ion impacts). For this purpose, the $\mathrm{S} / \mathrm{C}$ should be placed again on a condition of maximum illumination, thus along the Sun-asteroid line.

The autonomy of the GNC system is not always required. It follows from a trade-off between operational costs. system complexity and development costs of autonomous solutions that autonomy is only strictly required during the IBS Performance Phase and following. The operational concept, in autonomy sense, is summarised in Table 3.

\section{K GNC/AOCS modes and equipment}

A set of GNC/AOCS modes are proposed. Only operational modes are considered, i.e. excluding LEOP and commissioning phases. The mission phases and GNC modes matrix is shown in Table 4 . The equipment matrix is then shown in Table 5 . The required modes are:

- Safe mode (SM)

- Colliston Avoidance Mode (CAM): this mode is required to handle the risk of collision with the asteroid in case of a severe contingency. This mode is entered when CA monitoring flags an alarm.

- Inertial pointing mode (IPM): this mode implements inertial pointing and slew and unloading capabilities in order to point to different inertial directions:

- Inertial pointing sub-mode: used for taking images for Cruise and Approach navigation purposes (asteroid search and imaging). It is also used for communicating with ground.

- Wheel Off-loading sub-mode: used for regular off-loading of the wheels using chemical propulsion.

- Slew sub-mode: used to perform attitude manoeuvres.

- Asteroid-Pointing Mode (APM): it ensures (autonomous) pointing of the navigation/science camera and instruments towards the asteroid.

- Electrical Propulsion Control Mode (EPCM): used to perform the trajectory correction manoeuvres computed by ground.

- Orbit Control Mode (OCM): used in the asteroid close proximity operations. 
Table 2. In-orbit Operations Summary

\begin{tabular}{|c|c|c|c|c|c|c|}
\hline Phase & Sub-phase & $\begin{array}{l}\text { Asteroid } \\
\text { distance }\end{array}$ & Sub-phase description & $\begin{array}{l}\text { Duration } \\
\text { [days] }\end{array}$ & $\begin{array}{c}\text { Asteroid } \\
\text { size (NAC) } \\
\text { [pixel] }\end{array}$ & Sensors \\
\hline \multirow{3}{*}{$\begin{array}{l}\text { Approach } \\
\text { phase }\end{array}$} & Far approach & $\begin{array}{l}70000- \\
50 \mathrm{~km}\end{array}$ & $\begin{array}{l}\text { After asteroid detection, reach close } \\
\text { operations distance }\end{array}$ & 9 & $<1$ & NAC \\
\hline & Close approach & $50-5 \mathrm{~km}$ & $\begin{array}{l}\text { Start of proximity operations. } \\
\mathrm{P} / \mathrm{L} \text { commissioning and calibration. }\end{array}$ & 3 & $1-10$ & NAC \\
\hline & $\begin{array}{l}\text { Transition to far } \\
\text { characterisation }\end{array}$ & $\begin{array}{l}5 \mathrm{~km}- \\
500 \mathrm{~m}\end{array}$ & $\begin{array}{l}\text { Start of asteroid shape } \\
\text { characterisation. }\end{array}$ & 2 & $10-100$ & NAC \\
\hline \multirow{4}{*}{$\begin{array}{l}\text { Pre-IBS } \\
\text { performance } \\
\text { phase }\end{array}$} & IBS system test & $500 \mathrm{~m}$ & $\begin{array}{l}\text { Perform system tests for IBS phase } \\
\text { functioning mode (outside asteroid } \\
\text { Hill's radius) }\end{array}$ & 7 & 100 & $\begin{array}{l}\text { Ranging } \\
\text { / WAC }\end{array}$ \\
\hline & $\begin{array}{l}\text { Transition to } \\
\text { close } \\
\text { characterisation }\end{array}$ & $500-20 \mathrm{~m}$ & $\begin{array}{l}\text { Transition to close hovering position, } \\
\text { along Sun-Asteroid line for } \\
\text { illumination. }\end{array}$ & 7 & $>100$ & $\begin{array}{l}\text { Ranging } \\
\text { / WAC }\end{array}$ \\
\hline & $\begin{array}{l}\text { Close } \\
\text { characterisation }\end{array}$ & $20-50 \mathrm{~m}$ & $\begin{array}{l}\text { Asteroid detailed characterisation: } \\
\text { shape, rotation state, surface features, } \\
\text { gravity (radio science experiment). } \\
\text { Asteroid Orbit Determination. }\end{array}$ & 10 & $>100$ & $\begin{array}{l}\text { Ranging } \\
\text { /WAC }\end{array}$ \\
\hline & $\begin{array}{l}\text { Transition to } \\
\text { nominal } \\
\text { position }\end{array}$ & $20-50 m$ & $\begin{array}{l}\text { Acquisition of nominal Sun-asteroid- } \\
\text { S/C angle and asteroid distance. } \\
\text { Final system checks. }\end{array}$ & 4 & $>100$ & $\begin{array}{l}\text { Ranging } \\
\text { / WAC }\end{array}$ \\
\hline $\begin{array}{l}\text { IBS perf. } \\
\text { phase }\end{array}$ & IBS perf. phase & $10-15 \mathrm{~m}$ & Operation of the IBS & 180 & $>100$ & $\begin{array}{l}\text { Ranging } \\
\text { / WAC }\end{array}$ \\
\hline $\begin{array}{l}\text { Post-IBS } \\
\text { observation }\end{array}$ & $\begin{array}{l}\text { Post-IBS } \\
\text { observation }\end{array}$ & $20-50 \mathrm{~m}$ & $\begin{array}{l}\text { Acquisition of safe distance. } \\
\text { Asteroid Orbit Determination. }\end{array}$ & 30 & $>100$ & $\begin{array}{l}\text { Ranging } \\
\text { / WAC }\end{array}$ \\
\hline
\end{tabular}

Table 3. System autonomy during in-orbit phases

\begin{tabular}{|l|l|l|}
\hline \multicolumn{1}{|c|}{ Name of Phase } & \multicolumn{1}{c|}{ Orbit determination } & \multicolumn{1}{c|}{ Orbit manoeuvring } \\
\hline $\begin{array}{l}\text { LEOP, Escape and Commissioning } \\
\text { Phases }\end{array}$ & Ground based / Absolute & $\begin{array}{l}\text { Ground based / Lisa Pathfinder } \\
\text { Propulsion Module }\end{array}$ \\
\hline Cruise Phase & Ground based/Absolute & Electric Propulsion (EPS) operations \\
\hline Search Phase & Ground based/Absolute & EPS operations \\
\hline Approach Phase & Ground based/Absolute-Relative & EPS operations \\
\hline Pre-IBS Performance Phase & Ground based / Absolute-Relative & $\begin{array}{l}\text { EPS during non-autonomous sub- } \\
\text { phases } \\
\text { RCS during autonomous } \\
\text { (commissioning) sub-phases }\end{array}$ \\
\hline IBS Performance Phase & Onboard / Relative (commissioning) & Onboard / IBS + RCS \\
\hline Post-IBS Performance Phase & $\begin{array}{l}\text { Ground based/Absolute-Relative } \\
\text { (deflection estimation) }\end{array}$ & \begin{tabular}{l} 
RCS during autonomous sub-phases \\
\hline
\end{tabular} \\
\hline
\end{tabular}


Table 4. GNC/AOCS modes vs. mission phases

\begin{tabular}{|c|c|c|c|c|c|c|}
\hline Phase & SM & CAM & IPM & APM & ЕРCM & OCM \\
\hline Nominal transfer & \multirow{6}{*}{$(\mathrm{X})$} & & $\mathrm{X}$ & & $\mathrm{X}$ & \\
\hline NEO search & & & $\mathrm{X}$ & $\mathrm{X}$ & $\mathrm{X}$ & \\
\hline Approach & & & $\mathrm{x}$ & $\mathrm{X}$ & $\mathrm{X}$ & \\
\hline Pre IBS & & \multirow{3}{*}{$(\mathrm{X})$} & $x$ & $\mathrm{X}$ & $(\mathrm{X})$ & $\mathrm{X}$ \\
\hline IBS & & & $X$ & $X$ & & $\mathrm{X}$ \\
\hline Post IBS & & & $\mathrm{X}$ & $\mathrm{X}$ & & $\mathrm{X}$ \\
\hline
\end{tabular}

(X) only if required or commissioning

Table 5. Equipment vs. GNC/AOCS modes

\begin{tabular}{|c|c|c|c|c|c|c|c|c|}
\hline Phase & $\begin{array}{l}\text { Sun } \\
\text { Sensor }\end{array}$ & $\begin{array}{l}\text { STR } \\
\text { CHU }\end{array}$ & $\begin{array}{c}\text { Reaction } \\
\text { wheels }\end{array}$ & $\begin{array}{l}\text { Chemical } \\
\text { propulsion }\end{array}$ & $\begin{array}{c}\text { Electrical } \\
\text { propulsion }\end{array}$ & $\begin{array}{l}\text { Ranging } \\
\text { sensor }\end{array}$ & NAC & WAC \\
\hline SM & $\mathrm{X}$ & $(\mathrm{X})$ & (X) & $\mathrm{X}$ & & & & \\
\hline CAM & $\mathrm{X}$ & & & $\mathrm{X}$ & & $\mathrm{X}$ & & $\mathrm{X}$ \\
\hline IPM & & $\mathrm{X}$ & $\mathrm{X}$ & $(\mathrm{X})$ & & & Payload & \\
\hline APM & $(\mathrm{X})$ & $\mathrm{x}$ & $\mathrm{X}$ & & & $\mathrm{X}$ & \multicolumn{2}{|c|}{$\mathrm{X}$} \\
\hline EPCM & & $x$ & $\mathrm{X}$ & & $\mathrm{X}$ & & & \\
\hline $\mathrm{OCM}$ & $(\mathrm{X})$ & X & $\mathrm{X}$ & $\mathrm{X}$ & X & $\mathrm{X}$ & & $\mathrm{x}$ \\
\hline
\end{tabular}

(X) commissioning, reaction wheels unload or CA monitoring

\section{Asteroid Position and Velocity Estimation}

As pointed out in Ref. 8, in the pre- and post-deflection phase the position and (to a higher extent) the velocity of the asteroid centre of mass should be estimated with the highest possible precision to demonstrate that the artificially imposed deflection has effectively taken place. Such estimation could be based on ground observations (in the short periods when the asteroid is visible from Earth), or based on S/C ground tracking and relative asteroid-S/C metrology. The first approach could be only feasible in the very favourable case where the asteroid is seen from Earth, but this could only happen in very specific moments in the asteroid orbit not generally compatible with the mission. Furthermore, the derived precision will not be sufficient in the most general case. Therefore, the most practical solution will be to rely on the indirect measurement of the asteroid orbit by S/C flying in formation with the asteroid and determining the $\mathrm{S} / \mathrm{C}$ state with radio tracking means from Earth.

Due to its small size the asteroid cannot have the IBS orbiting around it without thruster corrections, which rules out an accurate measurement of the asteroid state with Doppler tracking alone while operating the low-thrust propulsion. Thus, it shall be preferred to define periods of time with no thrusting and flying at a safe (measurable) distance from the asteroid to enable the complete OD chain. This would be based on:

- Accurately determining the $\mathrm{S} / \mathrm{C}$ orbit

- Accurately determining the asteroid position wrt the $\mathrm{S} / \mathrm{C}$

A very rough approximation to the second activity could yield enough accuracy to the determination of the asteroid orbit.

For the first task we can already ascertain the achievable levels of knowledge in the OD process over the S/C. For that, we have made use of the Elecnor Deimos LOTNAV tool ${ }^{9}$ over the spacecraft trajectory to preview the achievable knowledge 1-sigma statistics under current OD assumptions (same as used for ExoMars ${ }^{10}$ ). Among other, the relevant cases are:

- Range and Doppler measurements from ESA's DSN, which includes stations at Cebreros, New Norcia and Malargüe

- As the previous case but adding DDOR from Cebreros-New Norcia baseline and from Cebreros-Malargüe baseline. 
Partial results of the covariance analysis OD simulations are provided in Figure 12. It is remarkable that there is a relative insensitivity to the number of ground stations in the results. It can be seen that for the cases without DDOR position error is stabilised after two days and velocity error after 10 days. When DDOR is included the stabilisation in position is obtained after the same amount of time, and in velocity is reduced to 4 days. Regarding the improvement in the obtained values, this goes further than one order of magnitude in position and half an order of magnitude in velocity. Given the fact that the deflection delta- $\mathrm{V}$ is implemented in the alongtrack direction, the relevant figure of merit is the one in that direction. Achievable values of error in alongtrack velocity are in the order of $2 \mathrm{~mm} / \mathrm{s}$ without DDOR and $0.4 \mathrm{~mm} / \mathrm{s}$ in velocity. Those values are roughly two-three orders of magnitude smaller than the deflection to measure, thus it shall be possible to measure the spacecraft alongtrack velocity with high precision even in the case of only one ground station.
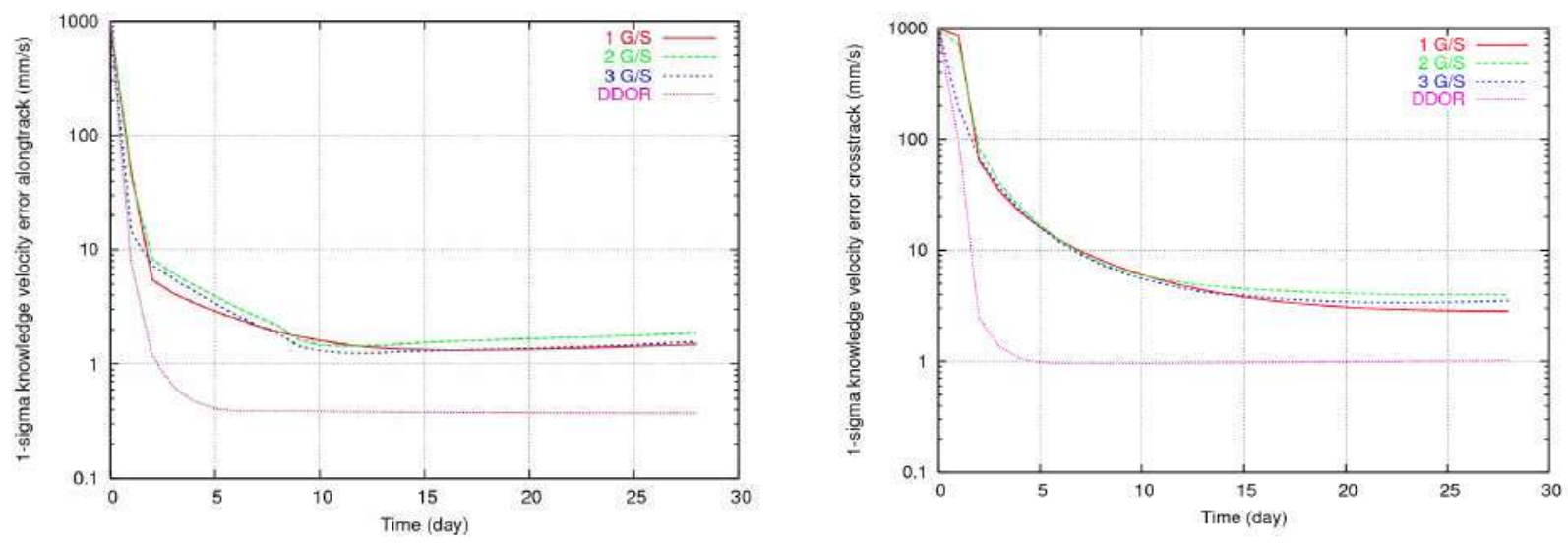

Figure 12. 1-sigma knowledge error in velocity for alongtrack (left) and crosstrack (right) state components after OD covariance analysis

\section{GNC design for Hovering phases and Collision Avoidance}

This section focuses on the GNC employed for the autonomous mission phases. The IBS performance (or Hovering) phase is characterised by the need to keep a certain distance with the asteroid in order to allow the ion beam to deflect its orbit from a design distance determined as optimum for this purpose. To this end, a navigation system must provide the GNC system with updated information about the relative position between the asteroid and the S/C. The considered technologies for IBS performance phase are then:

- Feature based navigation: complex solution, but does not need complete and precise mapping of the asteroid. Close proximity allows the identification of asteroid features to be tracked all along the operation during this phase. It becomes necessary when asteroid covers an angle greater than the FoV of the camera, or if asteroid covers a significant quantity of that FoV; in this case, asteroid of $4 \mathrm{~m}$ requires a FoV of $17^{\circ}$ in order to be completely observed by the camera. However, due to bad illumination conditions during all IBS performance (phase angle around $90^{\circ}$ ), this option has to be matured and validated in relevant scenarios.

- COB navigation: simple solution in mid ranges; not applicable for close proximity operations, in which asteroid size in camera FoV is too big. For this reason, if no WAC is available, this option is rejected as candidate. Conversely, if a WAC is available then this option is a good solution in terms of performance and complexity.

- Control Technologies: maintenance in a spherical control box, trying to keep distance and minimising $\Delta \mathrm{v}$ manoeuvres with good relative motion estimation accuracy is of paramount importance.

- Camera trade-off. regards the selection of a camera for the IBS performance phase. Assuming that the camera for search phase has a very narrow FoV, a new camera, answering to a different set of design requirements, should be selected for this phase.

- Altimeters / laser rangefinder trade-off. regards the selection of a range finder (altimeter/LIDAR) to solve the scale ambiguity of the camera.

A typical features Navigation algorithm (based on two frames) with altimetry support can be considered in order to evaluate performances. For a project scenario evaluated by Elecnor Deimos, relative position and velocity with 
respect to features can be estimated with accuracies in the order of $10 \mathrm{~cm}, 5 \mathrm{~mm} / \mathrm{s}$, which should make possible the effective control of the $\mathrm{S} / \mathrm{C}$ in a control box of \pm 1 metre around the nominal position.

\section{GNC Functional Architecture and Modes}

The functional architecture of the GNC subsystem during the Hovering phase is depicted in Figure 13. The following paragraphs describe the several S/W entities involved in the control loop. The employed equipment during Hovering phase is defined in Table 5.

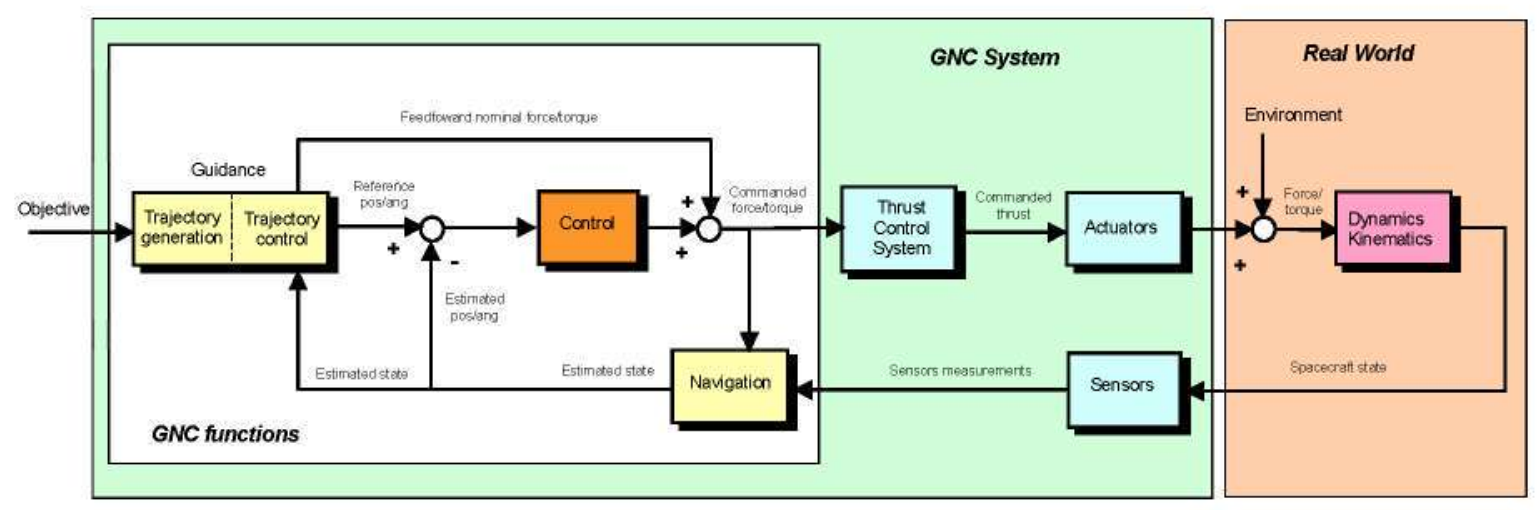

Figure 13. Functional GNC architecture for Hovering phase

- Attitude Guidance, Determination and Control (ADCS)

- Attitude guidance: in charge of computing the reference attitude profiles according to the operational mode

- Attitude determination: in charge of estimating the angular state of the SC (quaternion and angular rates)

- Attitude control: in charge of calculating commands that minimize the error between observed state and reference one

- Attitude sensors: set of equipment responsible of performing measurements to feed attitude determination with the purpose of obtaining SC angular state

- Attitude Actuators: set of equipment responsible of delivering the torque commands to the system. Thrusters (for reaction wheels desaturation and Collision Avoidance Manoeuvres) are foreseen along with reaction wheels (attitude control).

- Orbit Guidance Navigation and Control

- Guidance: in charge of computing of the reference profiles according to the operational mode and the feedforward commands

- Navigation: it is divided into Navigation Management which is in charge of managing planning and executing all Navigation-related activities and into Navigation Filter which is in charge of estimating the $\mathrm{SC}$ translational state of the SC (position and velocity are absolute during cruise navigation and become relative from approach navigation)

- Orbit control: in charge of calculating commands that minimize the error between observed state and reference one

- Thrust assignment system: it is a function responsible of distributing the commanded forces to the thrusters available. The objective is to minimise the fuel consumption and optimise thrusters employment

- Thrusters: set of equipment responsible of delivering commanded forces to the system

- Sensors: set of equipment responsible of performing measurements and taking images to feed navigation with the purpose of obtaining the best possible estimation of $\mathrm{S} / \mathrm{C}$ relative position and velocity with respect to the asteroid.

In what regards the Collision Detection and Avoidance, not clearly explicited so far, is a permanently active entity that monitors the $\mathrm{S} / \mathrm{C}$ state, and in case of collision risk it immediately signals the GNC/AOCS Manager to switch to CAM (see Section V-K). It is active in the IBS mission phase and can be summarised as:

- The $\mathrm{S} / \mathrm{C}$ performs the IBS experiment at about $10-20 \mathrm{~m}$ from the target 
- The S/C will be located at a phase angle of $90 \mathrm{deg}$

- Half of the asteroid will be visible to the S/C

- In contingency, the most secure option will be, as in the previous case, make use of the Sun sensor and make a CAM manoeuvre in the direction of the Sun

In the phases at shortest distances, the worst possible contingency would be an unbalance of the alongtrack thrust, in such a way that $\mathrm{S} / \mathrm{C}$ would be pushed against the asteroid. Assuming a $\mathrm{S} / \mathrm{C}$ weighted $400 \mathrm{~kg}$, with an unbalance of $15 \mathrm{mN}$, this would result in a collision after 13 minutes.

To cope with this situation, a grid of 8 thrusters with $1 \mathrm{~N}$ is proposed, such that at even detection and isolation, a manoeuvre would prevent the $\mathrm{S} / \mathrm{C}$ from effectively colliding against the asteroid. This manoeuvre. in any case, will be as simple as possible (just a jump backwards from nominal position. trying to impose some bigger distance between asteroid and $\mathrm{S} / \mathrm{C}$ ) and be as generic as possible to increase its robustness.

\section{GNC Programmatics and Technology Roadmap}

It is definitively a very challenging objective to place the spacecraft in an orbit $10 \mathrm{~m}$ "before" or over an asteroid of about $4 \mathrm{~m}$ in diameter and keep the position. This is even more challenging when at this close "hovering" position above the asteroids surface the two opposite solar electric propulsion thrusters are operated at maximum available thrust in order to apply an impulse transfer onto the asteroid. However, a NEO deflection demonstration mission is focused on just two scientific tasks: momentum transfer and observation. This results in a relatively simple system. since the spacecraft can mainly be based on technology with a high TRL. Therefore. the risk of the development of is considered low/medium, also when compared to other interplanetary missions.

Building a GNC system for interplanetary missions calls for an emphasis on the required autonomy of the mission. since the distances to the satellite and the close proximity operations with respect to the asteroid require the on-board capability of reacting autonomously without having to wait for ground support. These autonomy aspects must be traded off against the involved cost and the real necessity of that autonomy, since technological developments for autonomy are costly, in terms of time and resources.

The GNC sub-system is responsible to provide pointing and absolute/relative positioning observables and (when applicable) control for each mission phase, in nominal conditions but also in case of contingencies. The main GNC mission requirements and design drivers are to support the asteroid search phase by commanding the required manoeuvres to this end, to allow precise and safe in-orbit operations and support an accurate characterization of the asteroid target and, during all phases, control the satellite pointing with the required accuracy allowing the interplanetary navigation systems to work properly and reliably. While the pointing and stability specifications do not go beyond typical interplanetary mission requirements, there are challenging GNC aspects, namely:

- The high autonomy requirement during close proximity operations, aiming to being able to react without ground involvement

- The imprecise knowledge of target characteristics (shape, rotational state). which affects the navigation strategy during close proximity operations

- The closed-loop control of the spacecraft in hovering at $10 \mathrm{~m}$ from an asteroid of about $4 \mathrm{~m}$ in diameter. This is even more challenging when at this close "hovering" position above the asteroids surface the two opposite solar electric propulsion thrusters are operated at maximum available thrust (and associated imbalances)

The main technology gaps required for the feasibility of a NEO deflection mission can be grouped in two types:

- GNC and Image Processing systems

- Demonstrate the autonomous relative navigation for close proximity operations

- Demonstrate the autonomous execution of guidance manoeuvres

- Demonstrate the capability of autonomous shape and feature recognition

- Demonstrate the feasibility of camera plus altimeter measurement combination.

- Sensors

- Demonstrate the feasibility of finding a faint object with high sensitivity cameras.

Considering the current technological gaps. it is necessary to focus efforts on the autonomous determination and control of relative motion during hovering, and the following activities are beneficial:

- Involved sensors (cameras) can be tested in laboratory, since required relevant environment can be emulated in laboratory

- GNC technologies for operations close to the asteroid (including the IBS experiment) are more complex to test and validate on ground since they require a simulator of relative motion with all required degrees of freedom 
and control, which is not easy to reproduce in the lab. However, this solution must be pursued as much as possible to minimise cost.

\section{Acknowledgments}

The work presented in this paper was performed under the ESA SysNova R\&D Studies Competition for Innovation, with Technical University of Madrid as main contractor. and Elecnor Deimos. EADS Astrium GmbH and University of München as subcontractors.

\section{References}

'Bombardelli, C., and Peláez, J. "Sistema de modificación de la posición y actitud de cuerpos en órbita por medio de satélites guía". Patent number P20103035411, Presented at the Spanish Patent Office on March 11, 2010. PCT Patent Application PCTES2011/00001.

${ }^{2}$ Bombardelli C., et al "Active Removal of Space Debris - Ion Beam Shepherd for Contactless Debris Removal". ESA. Advanced Concepts Team, Ariadna Final Report (10-461 1c), 2011.

${ }^{3}$ Bombardelli, C., and Peláez, J. "Ion Beam Shepherd for Asteroid Deflection". Journal of Guidance. Control. and Dynamics, Vol. 34, No. 4, July-August 2011, pp. 1270-1272.

${ }^{4}$ Bombardelli, C., Urrutxua, H., Merino. M., Peláez. I., Ahedo, E., "The ion beam shepherd: A new concept for asteroid deflection". Acta Astronautica, Vol. 90, Issue 1, September 2013, pp. 98-102.

${ }^{5}$ NEODyS website: http:/newton,dm.unipi.jt/neodys/ or http//newton.dm. unipi.jt/neodys/

${ }^{\circ}$ JPL NEO program website: http://neo.jpl.nasa.gov/

"Cano, I.L., Sánchez, N.. Sánchez. M., Cornara, S., "Mission Analysis for the Don Quijote Phase A Study", IAC-07-A3.508. 58 th Congress of the International Astronautical Federation, Hyderabad. India. September 2007.

${ }^{8}$ Bombardelli, C. Urrutxua, H., Galvez, A., and Carnelli, I.,"The SIROCO Asteroid Deflection Demonstrator", AAS/AIAA Space Flight Mechanics Meeting Proceedings (AAS 12-227), 2012.

"Cano, J.L. et al., 'LOTNAV Tool, Software User's Manual", LOTNAV-DMS-TEC-SUM-30-E, Version 3.0, 03 Nov 2011.

${ }^{10}$ ExoMars Team at ESOC, ExoMars 2016 Mission: Consolidated Report on Mission Analysis. EXM-MS-RP-ESA-00008. issue $30,31 / 07 / 2012$. 